\title{
Non-linear Optimization of Parameters in Hill Kinetics
}

\author{
Željko Jeričević
}

\footnotetext{
a Department of Computer Engineering, Technical Faculty, Vukovarska 58, HR-51000 Rijeka, Croatia

b Department of Biology and Medical Genetics, Medical Faculty, Braće Branchetta 20, HR-51000 Rijeka, Croatia

Author's e-mail address: zeljko.jericevic@riteh.hr
}

RECEIVED: March 10, 2016 * REVISED: July 4, 2016 * ACCEPTED: July 5, 2016

Abstract: A novel least squares optimization of parameters in Hill enzyme kinetics has been developed. The method is based on transformation into "Michaelis-Menten like" type of equation with guaranteed convergence and the initial guess equal to zero. By using this approach, a three parameters non-linear optimization problem is transformed into a one-dimensional search for Hill's coefficient (exponential parameter $n$ ). Numerical experiments illustrating feasibility of the method and quality of the solution are presented.

Keywords: Hill, enzyme kinetics, non-linear, optimization, data processing, least squares.

\section{INTRODUCTION}

INEARIZATION of kinetic equations in order to determine kinetic parameters by the linear least squares is still in use for chemical and enzyme kinetics. However, linear optimization has serious drawbacks like redistribution of points and changing error statistics. Moreover, for the Hill model[1] linearization requires an initial guess for the parameter $V_{\max }$, which is an added complication.

\section{THEORY}

There are multiple forms of Hill kinetics. We will analyze the one described by the following equation which resembles Micheaelis-Menten equation but for the parameter $n$ :

$$
v=\frac{V_{\max } S^{n}}{K_{d}+S^{n}}
$$

where $S$ is a concentration of substrate, $v$ is velocity of reaction, $V_{\max }$ is saturation velocity, $K_{d}$ is apparent dissociation constant, and $n$ is Hill coefficient. Another form of Equation (1) has a $K_{A}$, which is ligand concentration at half saturation. The relationship between these two constants is $K_{d}=\left(K_{A}\right)^{n}$.
In order to determine $V_{\max }, K_{d}$, and $n$ from a collected series of measurements, $v$ as a function of $S$ is fitted to the model. Linearization transforms Equation (1) as follows:

$$
\begin{aligned}
& v K_{d}+v S^{n}=V_{\max } S^{n} \\
& v=\frac{\left(V_{\max }-v\right)}{K_{d}} S^{n} \\
& \log v=n \log S+\log \left(V_{\max }-v\right)-\log K_{d} \\
& \log \frac{v}{V_{\max }-v}=n \log S-\log K_{d}
\end{aligned}
$$

The Equation (5) shows that the intercept in this plot $\log \left(v /\left(V_{\max }-v\right)\right) v s . \log (S)$ is $-\log \left(K_{d}\right)$ with slope $n$. The construction of the plot requires an initial value for $V_{\max }$.

It is well known that linearization does not yield a correct estimate of parameters and strongly recommends the use of non-linear optimization techniques (see Wong ${ }^{[2]}$ for extensive discussion of this fact for Michaelis-Menten kinetics). However, he recommends general non-linear optimization routines which require a good initial guess and careful balancing of parameters. Jeričević and Kušter ${ }^{[3]}$ proposed a specialized nonlinear estimation method valid 
only for the Michaelis-Menten model. This proposed alternative method has guaranteed convergence with an initial guess of $K_{M}$ equal to zero and has no problem balancing the parameters. The main idea of the approach is to first reduce the dimensionality of the problem (by eliminating the linear parameter $V_{\max }$ from the normal equations) and then find the root of resulting non-linear equation. The work presented here is an application of a similar idea to the Hill equation.

Normal equations ${ }^{[4]}$ are developed using the least squares formalism:

$$
\sum_{i=1}^{N} \delta_{i}^{2}=\sum_{i=1}^{N}\left(v_{i}-{ }^{t h} v_{i}\right)^{2}=\sum_{i=1}^{N}\left(v_{i}-\frac{V_{\max } S_{i}^{n}}{K_{d}+S_{i}^{n}}\right)^{2}
$$

where $\delta_{i}$ is error between the experimental $v_{i}$ and theoretical ${ }^{t h} v_{i}$ value of dependent variable for experimental point $i$. The associated value of independent variable is $S_{i}$ and $N$ is number of experimental points.

Taking the derivative of the Equation (6) with respect to $V_{\max }$ (knowing it will be zero at the minimum) yields:

$$
\frac{\partial}{\partial V_{\max }} \sum_{i=1}^{N} \delta_{i}^{2}=-2 \sum_{i=1}^{N}\left(V_{i}-\frac{V_{\max } S_{i}^{n}}{K_{d}+S_{i}^{n}}\right) \frac{S_{i}^{n}}{K_{d}+S_{i}^{n}}=0
$$

Taking the derivative of the Equation (6) with respect to $K_{d}$ (again knowing it will be zero at the minimum) yields:

$$
\frac{\partial}{\partial K_{d}} \sum_{i=1}^{N} \delta_{i}^{2}=2 \sum_{i=1}^{N}\left(V_{i}-\frac{V_{\max } S_{i}^{n}}{K_{d}+S_{i}^{n}}\right) \frac{V_{\max } S_{i}^{n}}{\left(K_{d}+S_{i}^{n}\right)^{2}}=0
$$

Taking the derivative of the Equation (6) with respect to $n$ (again knowing it will be zero at the minimum) yields:

$$
\begin{aligned}
\frac{\partial}{\partial n} \sum_{i=1}^{N} \delta_{i}^{2}= & 2 \sum_{i=1}^{N}\left(V_{i}-\frac{V_{\max } S_{i}^{n}}{K_{d}+S_{i}^{n}}\right) \\
& \cdot\left(\left(\frac{S_{i}^{n}}{K_{d}+S_{i}^{n}}-1\right) \frac{V_{\max } S_{i}^{n} \ln S_{i}}{K_{d}+S_{i}^{n}}\right)=0
\end{aligned}
$$

Combining the Equations (7) with (8), and Equation (8) with (9) removes linear parameter $V_{\max }$ and yields two nonlinear equation in $K_{d}$ and $n$. By this procedure three parameters non-linear optimization problem is transformed into finding the solution of the system of two nonlinear equations: $f\left(K_{d}, n\right)$ shown as Equation (11) and $g\left(K_{d}, n\right)$ shown as Equation (13).

$$
V_{\max }=\frac{\sum_{i=1}^{N} \frac{v_{i} S_{i}^{n}}{K_{d}+S_{i}^{n}}}{\sum_{i=1}^{N} \frac{S_{i}^{2 n}}{\left(K_{d}+S_{i}^{n}\right)^{2}}}=\frac{\sum_{i=1}^{N} \frac{V_{i} S_{i}^{n}}{\left(K_{d}+S_{i}\right)^{2}}}{\sum_{i=1}^{N} \frac{S_{i}^{2 n}}{\left(K_{d}+S_{i}^{n}\right)^{3}}}
$$

$$
\begin{aligned}
& f\left(K_{d}, n\right)=\sum_{i=1}^{N} \frac{V_{i} S_{i}^{n}}{K_{d}+S_{i}^{n}} \sum_{i=1}^{N} \frac{S_{i}^{2 n}}{\left(K_{d}+S_{i}^{n}\right)^{3}} \\
& -\sum_{i=1}^{N} \frac{v_{i} S_{i}^{n}}{\left(K_{d}+S_{i}\right)^{2}} \sum_{i=1}^{N} \frac{S_{i}^{2 n}}{\left(K_{d}+S_{i}^{n}\right)^{2}}=0 \\
& V_{\max }=\frac{\sum_{i=1}^{N} \frac{v_{i} S_{i}^{n}}{K_{d}+S_{i}^{n}}}{\sum_{i=1}^{N} \frac{S_{i}^{2 n}}{\left(K_{d}+S_{i}^{n}\right)^{2}}}=\frac{\sum_{i=1}^{N}\left(\frac{S_{i}^{n}}{K_{d}+S_{i}^{n}}-1\right) \frac{v_{i} S_{i}^{n} \ln S_{i}}{K_{d}+S_{i}^{n}}}{\sum_{i=1}^{N}\left(\frac{S_{i}^{n}}{K_{d}+S_{i}^{n}}-1\right) \frac{S_{i}^{2 n} \ln S_{i}}{\left(K_{d}+S_{i}^{n}\right)^{2}}} \\
& g\left(K_{d}, n\right)=\sum_{i=1}^{N} \frac{v_{i} S_{i}^{n}}{K_{d}+S_{i}^{n}} \sum_{i=1}^{N}\left(\frac{S_{i}^{n}}{K_{d}+S_{i}^{n}}-1\right) \frac{S_{i}^{2 n} \ln S_{i}}{\left(K_{d}+S_{i}^{n}\right)^{2}} \\
& -\sum_{i=1}^{N}\left(\frac{S_{i}^{n}}{K_{d}+S_{i}^{n}}-1\right) \frac{v_{i} S_{i}^{n} \ln S_{i}}{K_{d}+S_{i}^{n}} \\
& \cdot \sum_{i=1}^{N} \frac{S_{i}^{2 n}}{\left(K_{d}+S_{i}^{n}\right)^{2}}=0
\end{aligned}
$$

The root can be found iteratively using NewtonRaphson metod ${ }^{[5]}$ and Jacobian matrix $J\left(K_{d}, n\right)$ shown as Equation (14):

$$
\begin{aligned}
& J\left(K_{d}, n\right)=\left[\begin{array}{ll}
\frac{\partial f\left(K_{d}, n\right)}{\partial K_{d}} & \frac{\partial f\left(K_{d}, n\right)}{\partial n} \\
\frac{\partial g\left(K_{d}, n\right)}{\partial K_{d}} & \frac{\partial g\left(K_{d}, n\right)}{\partial n}
\end{array}\right] \\
& \left(K_{d}\right)_{\text {new }}=K_{d}-\frac{1}{\left|J\left(K_{d}, n\right)\right|}\left|\begin{array}{ll}
f\left(K_{d}, n\right) & \frac{\partial f\left(K_{d}, n\right)}{\partial n} \\
g\left(K_{d}, n\right) & \frac{\partial g\left(K_{d}, n\right)}{\partial n}
\end{array}\right| \\
& n_{\text {new }}=n-\frac{1}{\left|J\left(K_{d}, n\right)\right| \mid}\left|\begin{array}{ll}
\frac{\partial f\left(K_{d}, n\right)}{\partial x} & f\left(K_{d}, n\right) \mid \\
\frac{\partial g\left(K_{d}, n\right)}{\partial x} & g\left(K_{d}, n\right)
\end{array}\right|
\end{aligned}
$$

Although the above system consisting of Equations (15) and (16) with good initial guess can be solved this way, it is much more complicated then the single equation used in Michaelis-Menten kinetics, repeated here for convenience:

$$
\begin{aligned}
f\left(K_{M}\right)= & \sum_{i=1}^{N} \frac{v_{i} S_{i}}{K_{M}+S_{i}} \sum_{i=1}^{N} \frac{S_{i}^{2}}{\left(K_{M}+S_{i}\right)^{3}} \\
& -\sum_{i=1}^{N} \frac{V_{i} S_{i}}{\left(K_{M}+S_{i}\right)^{2}} \sum_{i=1}^{N} \frac{S_{i}^{2}}{\left(K_{M}+S_{i}\right)^{2}}=0
\end{aligned}
$$

Besides of complexity for Hill equation, the more serious problem is a requirement for good initial guess for two parameters: $K_{d}$, and $n$. For that reason, we also developed a simpler approach, namely, for each guess of $n$, the system was transformed into Michaelis-Menten like one, 
using simple substitution $X=S^{n}$. That transforms the problem into systematic, stepwise one-dimensional search for optimal $n$, using guaranteed convergence of MichaelisMenten in each step for different guesses of $n$.

$$
\begin{aligned}
X=S^{n} & \\
f\left(K_{d}\right)= & \sum_{i=1}^{N} \frac{v_{i} X_{i}}{K_{d}+X_{i}} \sum_{i=1}^{N} \frac{X_{i}^{2}}{\left(K_{d}+X_{i}\right)^{3}} \\
& -\sum_{i=1}^{N} \frac{v_{i} X_{i}}{\left(K_{d}+X_{i}\right)^{2}} \sum_{i=1}^{N} \frac{X_{i}^{2}}{\left(K_{d}+X_{i}\right)^{2}}=0
\end{aligned}
$$

\section{RESULTS AND DISCUSSION}

Simulated data were constructed using different levels $(0$, 1,5 , and 10 percent) absolute error of $V_{\max }$ value. The noise was normally distributed with zero mean and added to the theoretical data. Each noise level was generated independently and then used to illustrate our procedure (see Table 1). The recovered and true values for parameters $K_{d}$ and $V_{\max }$ with error statistics ${ }^{[6]}$ are shown in Table 2. Graph of model data and simulated points with the corresponding noise levels used in Table 1 and 2 are shown in Figure 1.

Table 1. demonstrates that transforming Hill equation into Michaelis-Menten allows the least squares estimation of parameters $K_{d}$ and $V_{\max }$ for selected $n$. In other words, doing one-dimensional search for $n$ by optimizing Michaelis-Menten with guaranteed convergence in each step will yield the optimal value for $n$. The purist still may wish to use Newton-Raphson method for the few final improvement iterations. The Newton-Rapson method for the Hill equation as outlined in Equations (14-16) will converge if parameters $n$ and $K_{d}$ from one-dimensional search are used as initial guesses. At that point, the closeness to the root will assure the convergence of Newton-Raphson method.

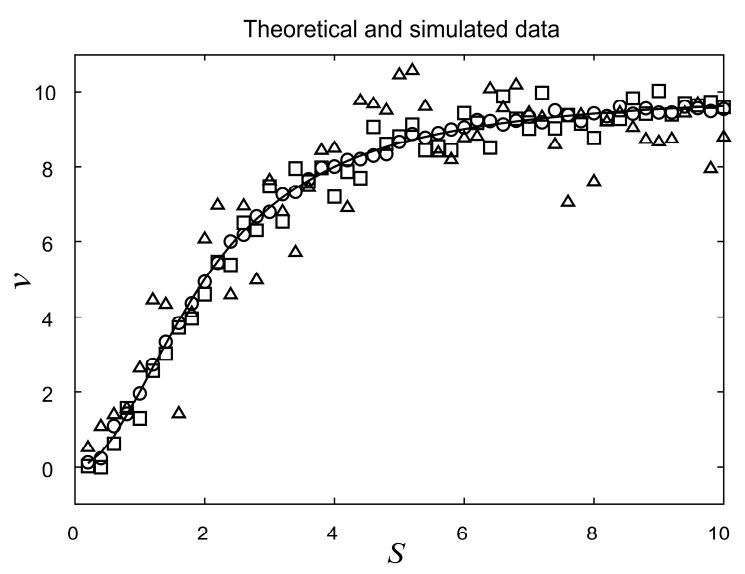

Figure 1. Plot of data summarized in Tables 1 and 2. Line is theoretical data $\left(n=2, K_{d}=4, V_{\max }=10\right)$. Circles are data with $1 \%$ error, squares are data with $5 \%$ error and triangles are data with $10 \%$ error.
Table 1 . The least squares sum for simulated data with various error levels. Three guesses for parameter $n$ are shown, true value is $n=2$ )

\begin{tabular}{crlr}
\hline \% error & $n=1.5$ & $n=2$ & $n=2.5$ \\
\hline 0 & 2.846 & $4 \times 10^{-10}$ & 1.905 \\
1 & 0.996 & 0.4906 & 2.423 \\
5 & 11.153 & 7.007 & 7.674 \\
10 & 57.202 & 53.886 & 55.462 \\
\hline
\end{tabular}

Table 2. Recovered parameters for $n=2$. True values are $K_{d}=4$ and $V_{\max }=10$

\begin{tabular}{cccccc}
\hline \% error & $K_{d}$ & error $K_{d}$ & $V_{\max }$ & error $V_{\max }$ & $\begin{array}{c}\text { Graph } \\
\text { symbol }\end{array}$ \\
\hline 0 & 4.000 & $2 \times 10^{-6}$ & 10.000 & $7 \times 10^{-7}$ & line \\
1 & 4.001 & 0.061 & 9.995 & 0.027 & circle \\
5 & 4.432 & 0.252 & 10.009 & 0.105 & square \\
10 & 3.262 & 0.544 & 9.623 & 0.263 & triangle \\
\hline
\end{tabular}

By combining a previously published ${ }^{[3]}$ method that has guaranteed convergence for optimizing MichaelisMenten kinetics with one dimensional search for the Hill coefficient $n$, our results show it is possible to construct a very robust and accurate non-linear optimization targeted to the Hill equation. The method also has the advantage of simplicity and speed. Guaranteed convergence algorithms are particularly important in analyzing large data sets from data intensive methods like sensor data. ${ }^{[7]}$

\section{CONCLUSION}

A specialized least squares algorithm for determining the parameters in the Hill equation using experimental measurement in enzyme kinetics was developed. The algorithm is simple, fast, and numerically stable. It transforms the nonlinear problem of optimizing three parameters into an efficient one dimensional search for Hill coefficient $n$.

\section{REFERENCES}

[1] A. V. Hill, J. Physiol. 1910, 40, iv.

[2] J. T-F. Wong, Kinetics of Enzyme Mechanisms, Academic Press, New York, 1975, p. 294.

[3] Ž. Jeričević, Ž. Kušter, Croat. Chem. Acta 2005, 78, 519.

[4] S. D. Conte, C. de Boor, Elementary Numerical Analysis, $3^{\text {rd }}$ Ed., McGraw-Hill, New York, 1980, p. 432.

[5] W. H. Press et al., Numerical Recipes, $3^{\text {rd }}$ Ed., Cambridge University Press, Cambridge, 2007, p. 1235.

[6] Y. Bard, Nonlinear Parameter Estimation, Academic Press, New York, 1974, p. 341.

[7] N. Nesakumar et al., J. Comp. Theor. Nanoscience 2014, 11, 2596. 